\title{
Mondes possibles, mondes fictionnels, mondes construits et processus de genèse
}

Daniel Ferrer

\section{(2) OpenEdition}

1 Journals

\section{Édition électronique}

URL : https://journals.openedition.org/genesis/127

DOI : $10.4000 /$ genesis. 127

ISSN : 2268-1590

\section{Éditeur :}

Presses universitaires de Paris Sorbonne (PUPS), Société internationale de génétique artistique littéraire et scientifique (SIGALES)

\section{Édition imprimée}

Date de publication : 20 juin 2010

Pagination : 109-130

ISBN : 978-2-84050-697-3

ISSN : 1167-5101

\section{Référence électronique}

Daniel Ferrer, «Mondes possibles, mondes fictionnels, mondes construits et processus de genèse », Genesis [En ligne], 30 | 2010, mis en ligne le 30 mai 2012, consulté le 30 mars 2023. URL : http:// journals.openedition.org/genesis/127 ; DOI : https://doi.org/10.4000/genesis.127 
Mondes possibles, mondes fictionnels, mondes construits et processus de genèse

Daniel Ferrer

O brave new world ShaKesPeARe, The Tempest J'ai désormais [...] tous les mondes dont j'ai besoin. ZolA, Ébauche de L'Argent

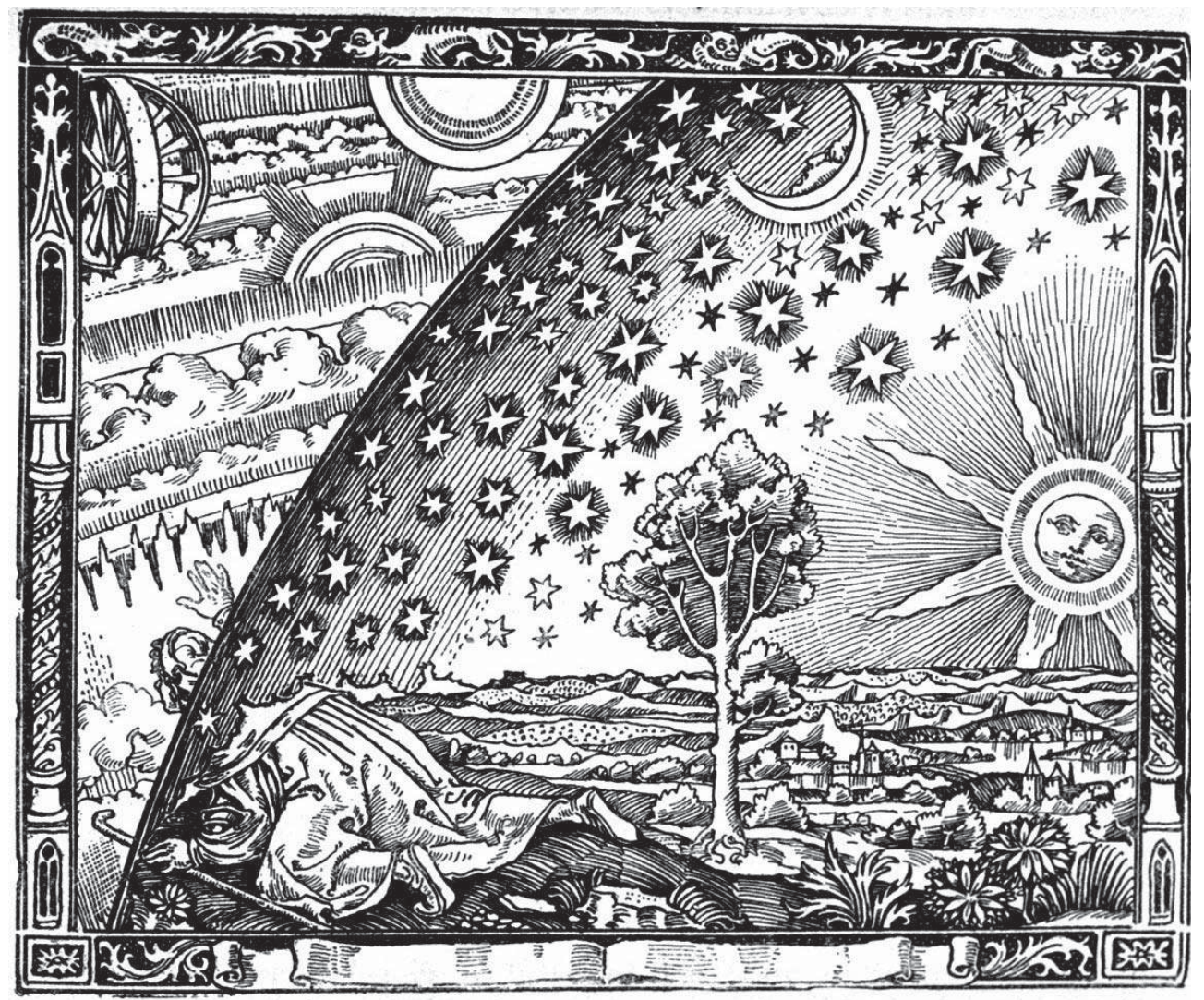

Dans un moment d'euphorie, la critique génétique pourrait prétendre incarner, au moins dans le domaine littéraire, ce que Leibniz appelle « la science de simple intelligence (qui regarde tous les possibles), où il faut enfin chercher la source des choses ${ }^{1}{ }^{\prime}$ et envisager de

1. Essais de Théodicée sur la bonté de Dieu, la liberté de l'homme et l'origine du mal, Paris, Flammarion, coll. «GF, 1969 , p. 360 . 
prendre pour modèle la grandiose construction que celui-ci place au point d'aboutissement de la Théodicée pour illustrer sa conception de la nécessité et sa théorie du meilleur des mondes possibles (celle dont Voltaire se gausse dans Candide). Le dispositif, « d'un brillant inconcevable et d'une grandeur immense », est mis en valeur par le franchissement d'une série de seuils imbriqués (un dialogue introduisant à une fable où un oracle ordonne un voyage et induit un rêve), avant d'être présenté par la déesse Athéna elle-même :

Vous voyez ici le palais des destinées dont j'ai la garde. Il y a des représentations, non seulement de ce qui arrive, mais encore de tout ce qui est possible ; et Jupiter en ayant fait la revue avant le commencement du monde existant, a digéré les possibilités en mondes, et a fait le choix du meilleur de tous. Il vient quelquefois visiter ces lieux pour se donner le plaisir de récapituler les choses et de renouveler son propre choix, où il ne peut manquer de se complaire. Je n'ai qu'à parler, et nous allons voir tout un monde que mon père pouvait produire, où se trouvera représenté tout ce qu'on en peut demander ; et par ce moyen on peut savoir encore ce qui arriverait, si telle ou telle possibilité devait exister (p. 362).

Cet édifice vertigineux est composé d'une infinité d'appartements dont chacun correspond à un monde alternatif. Visiter un de ces appartements permet d'apercevoir toute une vie " comme d'un coup d'œil, et comme dans une représentation de théâtre ». Chacun des appartements comporte par ailleurs «un grand volume d'écriture » racontant « l'histoire de ce monde $[\ldots]$ : c'est le livre de ses destinées ». Il suffit que l'on passe « dans un autre appartement, et voilà un autre monde, un autre livre », une autre destinée.

Les appartements allaient en pyramide; ils devenaient toujours plus beaux à mesure qu'on montait vers la pointe, et ils représentaient de plus beaux mondes. On vint enfin dans le suprême qui terminait la pyramide et qui était le plus beau de tous ; car la pyramide avait un commencement, mais on n'en voyait point la fin; elle avait une pointe, mais point de base ; elle allait croissant à l'infini. C'est [...] parce que entre une infinité de mondes possibles, il y a le meilleur de tous, autrement Dieu ne se serait point déterminé à en créer aucun ; mais il n'y en a aucun qui n'en ait encore de moins parfaits au-dessous de lui (p. 361).

Le Jupiter de Leibniz est à la fois créateur d'une œuvre et archiviste de sa propre création. C'est en termes esthétiques que se traduit l'évaluation de cette œuvre : le meilleur des mondes possibles est « le plus beau de tous » et s'incarne dans la plus belle des représentations. Comme dans le cas d'une genèse artistique ou littéraire, la perfection est essentiellement relative : une version l'emporte sur une autre parce qu'elle est meilleure qu'elle, mais elle est elle-même détrônée par une autre qui lui est supérieure, pour aboutir à un choix qui représente, du point de vue du créateur, un compromis, le meilleur possible. La perfection absolue n'est pas de ce monde, ni d'aucun autre envisageable, même par une intelligence absolue. Mais ce Jupiter est aussi un autogénéticien, qui se penche sur son propre avant-texte et l'érige en monument majestueux, socle de l'œuvre achevée. Cet agencement ressemble à une édition critique munie d'un appendice génétique qui collationnerait la totalité de l'avant-texte 2 . La perspective du Jupiter qui « vient quelquefois visiter ces lieux pour se donner le plaisir de

2. On pourrait parler d'édition hypermédia, d'une réalité virtuelle enrichie de liens numériques renvoyant à un texte d'où un simple clic permet de basculer vers une représentation visuelle (« Mettez le doigt sur la ligne qu'il vous plaira, [...] vous verrez représenté effectivement dans tout son détail ce que la ligne marque en gros », p. 361). 
récapituler les choses », comme d'ailleurs celle du visiteur qui parcourt les appartements superposés en les comparant au monde tel qu'il a effectivement été créé, sont semblables à celle du critique génétique qui contemple, d'un point de vue rétrospectif, l'œuvre achevée et l'ensemble des versions de cette œuvre.

Parler de visite des lieux, de parcours des appartements, de récapitulation, c'est situer l'investigation génétique au-delà d'une simultanéité idéale, dans une dimension temporelle cette dimension-là est, en revanche, totalement absente de la création elle-même. Il apparaît en effet que la création jupitéro-leibnizienne a toujours déjà eu lieu, qu'elle ne se déroule jamais, qu'elle est tout entière ramassée en un point d'origine. Est exclue toute possibilité d'accident, endogène ou exogène ${ }^{3}$, qui viendrait à l'improviste infléchir le cours de la création : tout est déjà prévu, achevé, avant même d'avoir commencé. Cet « avant-texte » exclut toute chronologie, ses versions sont données comme coprésentes, hiérarchisées en fonction d'un système de valeur préétabli et immuable (puisque Jupiter « ne pouvait manquer de choisir ce monde, qui surpasse en perfection tous les autres, qui fait la pointe de la pyramide »). S'il s'agissait d'une édition, il est clair qu'une telle édition n'aurait rien de génétique, et même qu'elle constituerait, malgré sa prise en compte de l'ensemble des versions, une dénégation radicale du processus génétique.

Est-ce à dire qu'il n'y a rien à retenir d'un tel modèle ? La notion de « mondes possibles » si brillamment instaurée par Leibniz a été source d'inspiration pour beaucoup : écrivains de science-fiction, philosophes, logiciens... Intuitivement, cette idée d'une pluralité de possibles représentés par des univers juxtaposés semble particulièrement adaptée aux études génétiques pour autant qu'elle ne soit pas conçue sur un mode statique.

Il est donc tentant de se tourner du côté de la « sémantique des mondes possibles » que les logiciens ont élaborée au $\mathrm{Xx}^{\mathrm{e}}$ siècle, à partir de la vision leibnizienne mais dans une perspective sensiblement divergente. Essayons de nous faire une idée, qui sera nécessairement très simplifiée et même un peu simpliste, d'une telle sémantique, pour voir si la critique génétique pourrait s'en inspirer de quelque façon ${ }^{4}$.

La logique traditionnelle a depuis longtemps mis au point tout un ensemble de procédures lui permettant d'évaluer la validité des propositions, mais elle ne sait pas bien comment traiter des propositions « modalisées », telles que celles ayant trait à la possibilité ou à la nécessité (« il est possible qu'on retrouve un jour le manuscrit d'Armance », « il est nécessaire qu'une œuvre ait un commencement »), les attitudes propositionnelles («Je crois que deux et deux peuvent faire cinq », « j'imagine qu'il pourrait venir ») ou encore les propositions dites « contrefactuelles » (« si les kangourous n'avaient pas de queue, ils tomberaient en avant », « si Joyce avait été moins myope, il serait peut-être devenu peintre plutôt qu'écrivain », « si je m'étais retourné, je l'aurais vu »), qui décrivent des états de choses non conformes à la réalité.

3. Voir Daniel Ferrer, «L'écriture et l'accident », dans Olga Anokhina et Sabine Pétillon (dir.), Critique génétique : concepts, méthodes, outils, Paris, IMEC éditeur, coll. «Inventaires », 2009.

4. J'avais fait une suggestion en ce sens dans « La toque de Clementis : rétroaction et rémanence dans les processus génétiques » (Genesis, $n^{\circ}$ 6, «Enjeux critiques », 1994), puis, plus explicitement, dans « Le matériel et le virtuel : du paradigme indiciaire à la logique des mondes possibles », Pourquoi la critique génétique? Méthodes, théories, dir. Michel Contat et Daniel Ferrer, Paris, CNRS Éditions, 1998. Ces suggestions sont quelque peu développées dans le présent article, toujours de manière programmatique.
Logique modale et mondes possibles 
Le traitement logique de telles propositions relève d'une logique modale. La sémantique des mondes possibles a été inventée dans ce cadre, pour résoudre des difficultés intensionnelles en les transposant sur un plan extensionnel, c'est-à-dire en transformant ces problèmes qui relèvent de la signification des propositions en problèmes de référence. Plus concrètement, il s'agit d'incarner les modalités dans des mondes postulés (un monde où le manuscrit d'Armance ressurgirait, le monde de mes croyances, un monde de kangourous sans queue...). Dans ce cadre, on dira qu'une proposition est possible s'il existe au moins un monde, accessible à partir du monde actuel, dans lequel cette proposition est vraie, tandis qu'une proposition est nécessaire si elle est vraie dans tous les mondes accessibles à partir du monde actuel.

Sont ainsi définis abstraitement un ensemble de mondes gravitant les uns autour des autres, régulés par la notion d'accessibilité. Tout dépend de ce qu'on range sous cette appellation. Le plus simple est de considérer comme accessible à partir du monde actuel tout monde qui respecte la loi de non-contradiction et de définir ces mondes comme compossibles. Mais le monde dans lequel nous vivons ne sera pas nécessairement le monde de référence choisi et rien n'empêche de définir des règles d'accessibilité plus complexes (nous y reviendrons).

En ce qui concerne le statut ontologique accordé par les philosophes à ces mondes imaginés par la logique, plusieurs conceptions s'opposent parmi lesquelles on peut distinguer trois lignes principales. La plus répandue, et la plus conforme au sens commun, est souvent qualifiée de « réalisme modéré » : c'est l'idée que le possible fait partie de la réalité, que les mondes possibles sont inséparables du monde actuel, auquel ils appartiennent à divers titres, constructions mentales ou virtualités logiques. Une deuxième position est celle de l'« antiréalisme », qui nie toute pertinence aux mondes possibles et déconstruit même, dans le cas de Goodman, la notion de possibilité5. Dans cette perspective, nous ne disposons que de versions du monde, et nous n'avons aucun moyen de dire que l'une d'entre elles correspondrait à une réalité indépendante de toute version 6 . Enfin une conception diamétralement opposée défend une forme radicale du réalisme, c'est le « réalisme modal » de David Lewis ${ }^{7}$, pour lequel tous les mondes possibles existent au même titre que le monde actuel - au point que la notion même de monde actuel doit être considérée, selon cette conception, non pas comme un absolu, mais comme une notion indexicale, désignant le monde, quel qu'il soit, du point de vue duquel on se place. Nous allons voir que ces trois conceptions peuvent avoir chacune leur intérêt pour la critique génétique qui, dans sa recherche de modèles féconds, n'a nullement besoin de prendre parti entre elles.

\footnotetext{
5. Il parle de «trépas du possible » (Faits, fictions et prédictions, Paris, Éditions de Minuit, Paris, 1985, p. 74). La notion de possible est remplacée par celle de disposition : au lieu de dire « il est possible que ce chapitre soit développé », Goodman dirait « ce chapitre est développable », ce qui lui permet d'éviter de faire référence à un état de choses fictif et de la remplacer par une description de la réalité. À première vue, cette voie ne paraît pas particulièrement prometteuse pour la critique génétique. Sans prétendre être plus nominaliste que le roi, on peut remarquer naïvement que, jusqu'à ce que Joyce se mette à le déformer, le langage de la fiction n'était pas considéré comme déformable. Ce que le généticien recherche, c'est un moyen de penser le surgissement de ce nouveau prédicat dispositionnel.

6. Voir notamment Of Mind and Other Matters, Cambridge, Harvard University Press, 1984, p. 125.

7. Counterfactuals, Cambridge, Harvard University Press, 1973.
} 
La notion de monde possible ayant permis de remarquables avancées sur un certain nombre de questions, elle a connu un renouveau d'intérêt parmi les philosophes, mais aussi dans de nombreuses autres disciplines ${ }^{8}$. On s'est toutefois bientôt rendu compte que l'emploi qui en était fait, y compris en philosophie, était souvent très éloigné de l'usage technique qu'en faisaient les logiciens, et qu'il relevait en général de l'analogie plutôt que de l'application stricte (ce qui n'est pas un défaut, à condition que tout le monde en soit conscient et surtout que l'analogie éclaire plus qu'elle n'obscurcisse). Soulignons qu'il s'agit avant tout d'une manière de poser les problèmes.

Il semble tout à fait naturel de rapprocher la théorie des mondes possibles de celle de la fiction. On peut par exemple essayer d'assimiler le statut de la fiction à celui des propositions modalisées ou à celui des propositions contrefactuelles mentionnées ci-dessus. La logique traditionnelle est tout aussi impuissante à traiter les propositions portant sur les œuvres de fiction : elle ne sait pas leur assigner une valeur de vérité ou bien elle considère qu'elles sont uniformément fausses 9 . On a suggéré que la fiction reposait sur un «énallage de modalité », c'est-à-dire que l'indicatif qu'elle emploie couramment remplacerait un conditionnel ${ }^{10}$. Les situations contrefactuelles, de leur côté, ne constituent-elles pas de petits romans, peuplés de créatures fictionnelles (kangourous sans queue, Joyce sans lunettes, un moi qui se serait retourné et qui aurait vu)?

Un tel rapprochement ouvre de nombreuses pistes, mais il soulève aussi de nombreuses difficultés, qui ont été recensées systématiquement par Ruth Ronen ${ }^{11}$. Les débats sur la question sont toujours vivaces ${ }^{12}$, mais on considère généralement que le rapprochement est justifié par la fécondité des avancées théoriques qu'il a permises ${ }^{13}$. Nous n'avons pas la place de discuter ici l'ensemble de ces avancées, ni les objections de fond qu'elles peuvent soulever. Les unes et les autres ne nous concernent que dans la mesure où elles ont des conséquences pour une théorie de la genèse.

8. « Possible Worlds in Humanities », Arts and Sciences, Proceedings of Nobel Symposium, 65, Sture Allen (dir.) Berlin, New York, Walter de Gruyter, 1989.

9. Et pourtant, comme le fait remarquer Umberto Eco (The Limits of Interpretation, Bloomington, Indiana University Press, 1994, p. 64), nous n'hésiterions pas à recaler un étudiant de littérature qui ne saurait pas dire, par exemple, que la proposition «Desdémone est fidèle » est vraie et que la proposition «Emma Bovary est fidèle » est fausse. 10. Catherine Kerbrat-Orecchioni, dans son article précurseur « Le texte littéraire : non-référence, auto-référence ou référence fictionnelle », Texte, 1982, p. 38.

11. Possible Worlds in Literary Theory, Cambridge, Cambridge University Press, 1994, passim.

12. En 2005-2006, un riche séminaire a été consacré à « La théorie des mondes possibles : un outil pour l'analyse littéraire ? », sous la responsabilité de Françoise Lavocat. Les résumés sont consultables en ligne sur <www.fabula. org/atelier.php?Th\%26eacute\%3Borie_des_mondes_possibles\%3A_un_outil_pour_1\%27analyse_ litt\%26eacute\%3Braire\%3F>.

13. Parmi une abondante bibliographie, on dispose, en français, de Umberto Eco, Lector in Fabula ou la Coopération interprétative dans les textes narratifs, Paris, Grasset, 1985, et Thomas Pavel, Univers de la fiction, Paris, Éditions du Seuil, 1988. Il faut lire par ailleurs Marie-Laure Ryan, Possible Worlds, Artificial Intelligence and Narrative Theory, Bloomington, Indiana University Press, 1991, et Lubomir Doležel, Heterocosmica. Fiction and Possible Worlds, Baltimore, The Johns Hopkins University Press, 1998. On a la chance de trouver en ligne une très lucide introduction de Françoise Lavocat : «L'œuvre littéraire est-elle un monde possible ? »<www. fabula.org/atelier.php?L\%27oeuvre_litt\%26eacute\%3Braire_est\%2Delle_un_monde_possible\%3F\#_ ednref42>. 


\section{Mondes comparés et mondes stipulés}

Avant d'en discuter, deux préalables méthodologiques doivent être posés. La notion de monde possible est invoquée dans des contextes très divers, mais, comme le fait remarquer Eco, elle n'a d'utilité que si l'on veut mettre en parallèle et comparer plusieurs états de choses, monde actuel et monde possible ou mondes possibles entre eux ${ }^{14}$. Deuxièmement, il ne faut pas perdre de vue que les mondes possibles sont des constructions. Ce ne sont pas des univers observables, telles des planètes lointaines contemplées à partir du monde actuel au moyen de puissants instruments d'optique, ce sont des mondes stipulés ${ }^{15}$.

La première de ces mises en garde doit plutôt être prise comme un encouragement pour la critique génétique. On sait bien que la méthode génétique a précisément pour base la comparaison : comparaison d'états avant-textuels et de leurs différences verbales, mais aussi, nous allons y revenir, comparaison des significations produites par ces versions 16 et comparaison de mondes décrits par ces formes verbales et présentés à travers ces significations.

Le deuxième préalable soulève des problèmes beaucoup plus difficiles, mais pourra être également considéré comme favorable à l'approche génétique. On dira volontiers, avec les principaux théoriciens de la fiction, que les mondes fictionnels sont des objets construits : ils sont engendrés par le texte des œuvres qui font mine de les décrire, mais qui, de fait, les stipulent (il n'existe pas de Maison Usher préexistant à la description qu'en fait Poe). On pourrait penser, en revanche, que les objets de la critique génétique sont des objets observés et non construits : le généticien observe des documents, des versions textuelles qu'il a sous les yeux (ou qu'il s'efforce de reconstituer par inférence si les documents sont manquants). Alors que l'historien doit constamment bâtir des scénarios contrefactuels pour comprendre ce qui s'est réellement passé17, le généticien (dans un premier temps) n'a qu'à découvrir des possibles actualisés sur le papier.

Comme les mondes de Leibniz dans la conscience divine, les mondes de l'avant-texte coexistent dans les manuscrits. Ils sont accessibles par l'intermédiaire du travail philologique, qui joue le rôle intercesseur d'Athéna. On peut les visiter, les contempler, fût-ce au moyen d'un

14. Lector in Fabula, op. cit., p. 69.

15. «Un monde possible n'est pas un pays lointain qu'on rencontre sur son chemin ou qu'on regarde au télescope. En général, un monde possible différent du nôtre est trop éloigné : même si nous voyagions plus rapidement que la lumière, nous ne pourrions pas l'atteindre. Un monde possible est donné par les conditions descriptives que nous lui associons. [...] Les "mondes possibles" sont stipulés, ils ne sont pas découverts au moyen de puissants télescopes », Saul Kripke, La Logique des noms propres (Naming and Necessity), Paris, Éditions de Minuit, 1982 , p. 32.

16. Doležel parle à cet égard de monde intensionnel, par opposition au monde extensionnel (« Extensional and Intensional Narrative Worlds », Poetics, $\left.n^{\circ} 8,1979\right)$. S'il est important, en genèse comme en sémantique, de distinguer le sens (intension, «mode de donation de la référence ») et la référence (extension), on ne voit guère, pour le coup, l'intérêt d'utiliser le concept de monde. Si mondes intensionnels il y a, ils sont directement corrélés à la forme de l'expression (à ce que nous appellerons ci-dessous les mondes textuels), ils ne jouissent pas de l'autonomie (au moins partielle) dont jouissent les mondes extensionnels.

17. Voir Paul Veyne, Comment on écrit l' histoire, suivi de Foucault révolutionne l' histoire, Paris, Éditions du Seuil, 1979, p. 78-79. Plus généralement, «In the concept of an action is [...] implicit a comparison or contrast between a state of affairs resulting from the action and another state which would otherwise, i.e. had it not been for the performance of the action, have obtained [...] That which "would have otherwise have obtained" never comes true (occurs). It is "contrary to fact”" (Georg Henrik von Wright, Causality and Determinism, New York, Columbia University Press, 1974, p. 41). 
instrument d'optique (la loupe du chercheur en manuscrits). Mais contrairement aux mondes leibniziens qui existent de toute éternité, au point que le Créateur lui-même les découvre plus qu'il ne les crée, les mondes de l'avant-texte sont bien des mondes stipulés. Quelle que soit la forme grammaticale qu'ils revêtent, les manuscrits de travail peuvent être analysés comme des prescriptions 18 : ils sont des directives, visant en dernier ressort à la construction du texte final. Il faut préciser que cette construction s'opère à travers la stipulation d'une série d'étapes textuelles intermédiaires (les approximations successives de la formulation définitive), mais aussi à travers la construction progressive du monde auquel renverra le texte final, construction qui prend elle-même la forme d'une stipulation de mondes intermédiaires (permettant l'élaboration par étapes d'éléments tels que les personnages et le décor).

Deux types de mondes possibles s'offrent donc à la comparaison dans le cadre de la genèse : des mondes textuels et des mondes fictionnels ${ }^{19}$. Avec ce que nous appellerons les mondes textuels, il s'agit des mots, des phrases et de leur disposition sur le papier, des formulations et des techniques littéraires : ce sont les versions alternatives de configurations textuelles ou narratives que projette l'avant-texte. Ainsi, en tête du premier Plan de Madame Bovary (fo 1 , ms. gg 9, Bibliothèque municipale de Rouen), deux versions de l'œuvre sont projetées, deux univers textuels virtuels sont confrontés, l'un s'inscrivant en première ligne du bloc d'écriture principal (« Charles Bovary officier de santé 33 ans quand commence le livre veuf déjà d'une femme plus vieille que lui »), l'autre dans un ajout marginal («Commencer par son entrée au collège ») : soit un roman qui commencerait alors que Charles est adulte et un autre qui commencerait alors qu'il est adolescent.

\section{Mondes textuels et mondes fictifs}

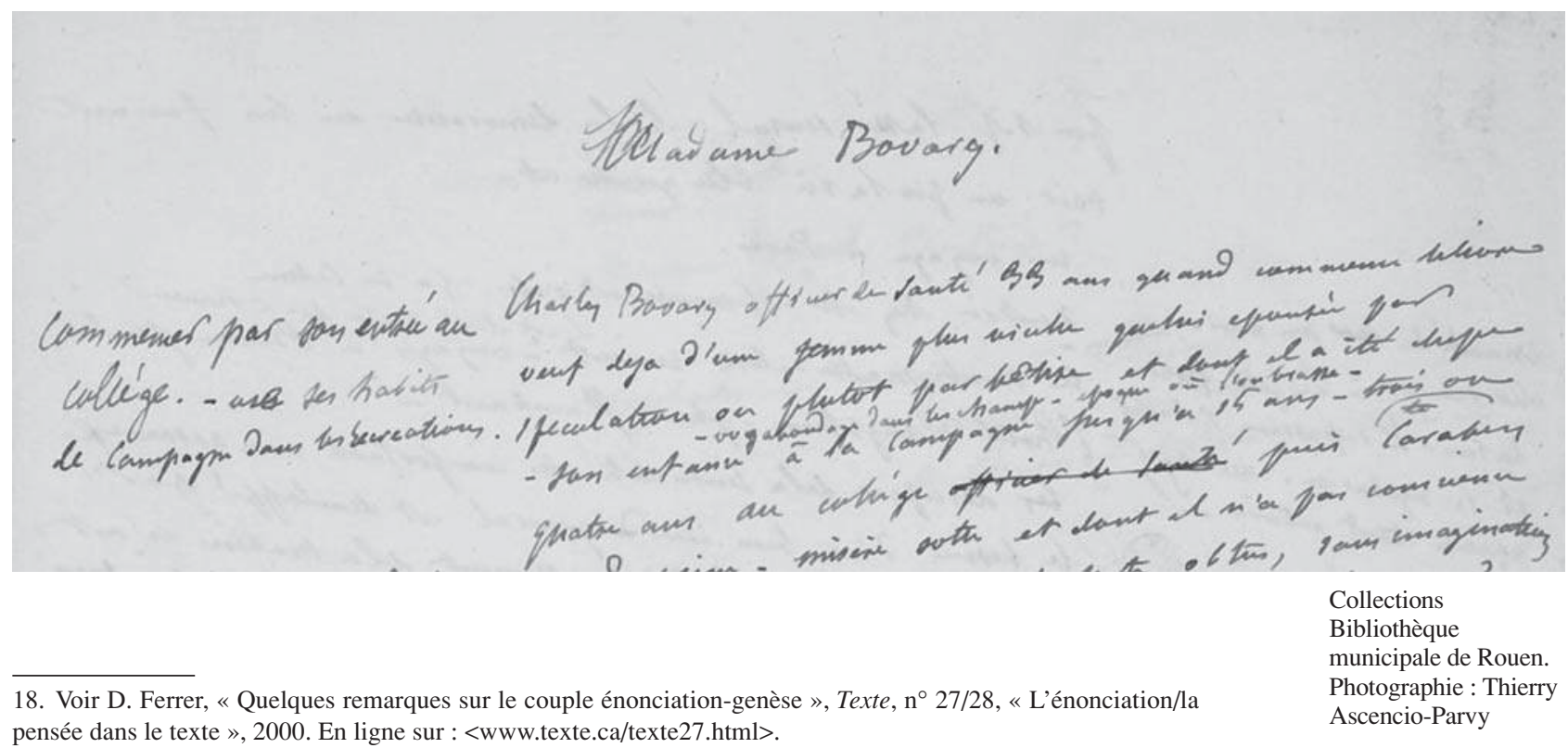
pensée dans le texte », 2000. En ligne sur : <www.texte.ca/texte27.html>.

19. Dans cet article, la plupart des exemples sont empruntés au genre romanesque, mais les mondes fictionnels ne sont pas réservés à ce qu'on appelle généralement la fiction. Contrairement à ce qu'on pourrait croire, ils jouent notamment un rôle important dans la genèse de la poésie. 
La première solution n'impliquait pas un univers fictif différent : la jeunesse de Charles aurait pu être racontée par des retours en arrière, ou bien elle aurait été laissée dans l'ombre (voir ci-dessous la section « Incomplétude ? »), mais il n'y a pas de raison particulière de penser qu'elle aurait été substantiellement différente. Ce sont bien deux mondes textuels qui sont en jeu. Pour être tout à fait clair, on prendra un autre exemple qui s'en tient strictement à la formulation : l'alternative entre un monde textuel dont la dernière phrase contiendrait les mots « un gigantesque perroquet » (premier brouillon d'Un cour simple, BnF, ms., nafr, 23663-349 $\mathrm{v}^{\circ}$ ) et un monde qui comporterait (comme les versions suivantes) « un perroquet gigantesque ».

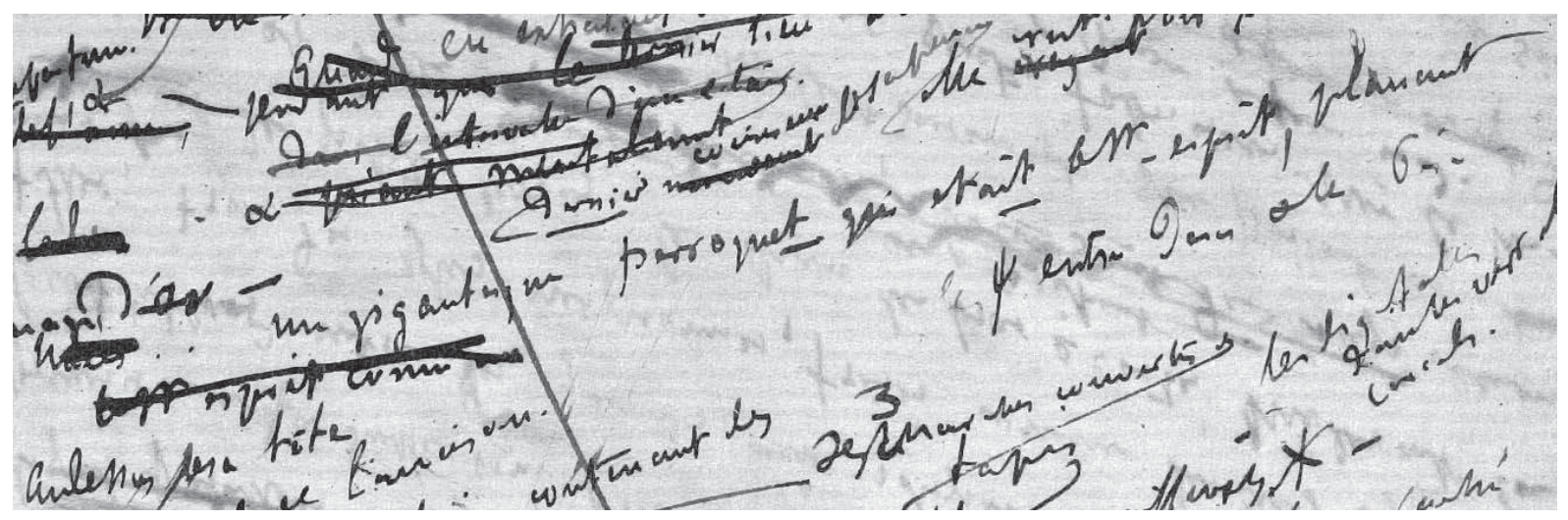

Y a-t-il un intérêt quelconque à invoquer ici la notion de monde possible ? Cette notion nous rappelle d'abord qu'en confrontant les mots inscrits sur les manuscrits, nous confrontons en fait des œuvres virtuelles, stipulées par ces mots. Elle nous permettra aussi de faire appel à la notion d'accessibilité. Enfin, comme j'ai essayé de le montrer ailleurs ${ }^{20}$, c'est la notion d'identité à travers les mondes possibles qui permet de résoudre, en s'appuyant sur l'idée de désignateur rigide, la question de l'identité de l'œuvre à travers ses versions (comment peuton dire qu'un récit qui commence avec la trente-troisième année de Charles Bovary est une version du célèbre texte qui débute avec les mots « Nous étions à l'Étude, quand le Proviseur entra, suivi d'un nouveau habillé en bourgeois... » ?) et, par là même, celle de la définition de l'avant-texte.

L'avantage qu'il y a à confronter des mondes fictionnels dans le cadre de la genèse est plus facile à percevoir. Mais il faut d'abord préciser ce qu'on entend par monde fictionnel.

Certains théoriciens ont dénié explicitement à l'œuvre de fiction tout pouvoir de référence, prétendant la réduire aux phrases qui la composent, ou encore à un jeu d'écho intertextuel. Mais beaucoup plus nombreux sont les critiques qui, sans formuler une telle dénégation, ont ignoré en pratique les problèmes de référence. La critique génétique elle-même a déployé beaucoup d'énergie pour explorer la genèse des formes ou des significations, mais elle a souvent considéré avec moins d'attention la genèse des mondes fictifs. Question de période, sans doute. Dans les années qui ont vu le développement de la discipline, la référence

20. « La toque de Clementis... », art. cit., p. 98-100. 
constituait un point aveugle 21 ou même un tabou dans les études littéraires en général 22 et il était par ailleurs tout particulièrement important de se démarquer de la vision traditionnelle de la genèse comme processus mimétique de transposition de la réalité dans l'œuvre. Pourtant la génétique textuelle ne peut se permettre de contourner élégamment la question, comme le font d'autres formes de critique, car la référence joue un rôle important dans le processus de fabrication des textes.

Du côté de la lecture, il est clair que c'est le texte de l'œuvre qui engendre le monde fictif, le récit qui produit la diégèse (voir «Appendice terminologique »), mais du point de vue de l'écriture, de l'engendrement effectif de l'œuvre, les choses sont beaucoup plus complexes 23 . Il est vrai que, dans certains cas, la référence apparaît comme une sorte de sous-produit. Ainsi, sur le premier brouillon de l'épisode des «Sirènes » dans Ulysses, Joyce utilise les mots «crushed strawberry » pour décrire la robe de la vice-reine. Dès le second brouillon, cette couleur est remplacée par une autre : « eau-de-Nil ». Le changement semble motivé par une question de rythme, mais aussi de sens : les connotations des deux termes sont différentes, et l'appellation « eau-de-Nil » vient saturer un paradigme aquatique très important et qui se renforce au fil des versions. Cependant, il en résulte bien deux univers fictifs distincts : l'un où la vice-reine est vêtue de rouge, l'autre où elle est vêtue de vert.

Un autre exemple est d'autant plus significatif qu'il porte sur un poème nonsensique, The Walrus and the Carpenter, qui s'attaque délibérément au processus de référence. Lewis Caroll révèle qu'il avait proposé à son illustrateur Tenniel, pour faciliter sa tâche, de remplacer le personnage du carpenter (menuisier) par un baronet ou même un butterfly (papillon), ou par tout autre être dont l'appellation serait prosodiquement équivalente. C'est dire clairement que la référence est une conséquence accessoire, une simple retombée du choix des mots, mais c'est en même temps consacrer son importance en se déclarant prêt à infléchir le texte pour la commodité de l'illustration qui la fixera ${ }^{24}$.

21. Pour prendre l'exemple emblématique de Barthes, il est intéressant de constater que, face au manuscrit, l'image de mondes pluriels lui venait naturellement sous la plume, mais que, dressant l'inventaire de ces mondes, il évite complètement les mondes représentés (qu'on ne peut pas réduire à un « sens associé ») : «J'ai devant moi une page de manuscrit ; quelque chose [...] qu'on appelle la lecture, se met en marche. Cette lecture, où vais-je, où puis-je l'arrêter? Certes, je vois bien de quel espace mon œil part ; mais vers quoi ? Sur quel autre espace accommode-t-il ? va-t-il derrière le papier? (mais derrière le papier, il y a la table). Quels sont les plans que toute lecture découvre ? Comment est construite la cosmogonie que ce simple regard postule ? Singulier cosmonaute, je traverse bien des mondes, sans m'arrêter à aucun d'eux : la blancheur du papier, la forme des signes, la figure des mots, les règles de la langue, les contraintes du message, la profusion des sens associés », R. Barthes, Variations sur l'écriture "Infini », Euvres complètes, vol. III, p. 1564. Cité par Jean-Louis Lebrave, "La genèse de La Chambre claire », Genesis, $\mathrm{n}^{\circ}$ 19, « Roland Barthes », 2002.

22. Voir 1'article déjà cité de Catherine Kerbrat-Orecchioni, «Le texte littéraire : non-référence... ».

23. Doležel pose une distinction absolue entre ce qu'il appelle les I-texts (world imaging texts) dont la fonction est de représenter le monde actuel, qui leur préexiste nécessairement, et les C-texts (world constructing texts) dont la fonction est de construire des mondes qui n'ont pas d'existence préalable. Il s'oppose en cela aux constructivistes radicaux, comme Goodman, pour qui tous les mondes sont construits, au moyen de mots ou d'autres symboles. Pour Doležel, l'image du monde décrit par les I-texts est constamment remaniée, dans un processus de validation et de réfutation, tandis que le monde fictionnel est fixé « once its creator has fixed the constructing text » (Heterocosmica, op. cit., p. 26). Il n'est pas certain que les mondes fictionnels dont l'écriture est achevée soient de fait aussi fixes qu'il veut bien le dire, mais en tout cas, dans le cadre de la genèse, rien n'est fixé et tout bouge à la fois. 24. Voir l'article de Christine Collière, «Illustrating the Sylvie and Bruno Books: the Collaboration of Lewis Carroll and Harry Furniss », Recto Verso, n ${ }^{\circ} 3$ <www.revuerectoverso.com/spip.php?article90>. 
Dans d'autres cas, sans doute plus nombreux, la référence est première et c'est elle qui va servir de base à la genèse. Quand Flaubert inscrit, sur le scénario d'ensemble initial d'Un cœur simple, "vision mystique. Son perroquet est le St Esprit », ces mots ne visent pas directement la formulation définitive (ils n'apparaissent jamais dans le texte final, ni même dans aucun des brouillons) mais la situation (mentale, en l'occurrence) à laquelle renverra le texte, un état du monde fictif que décrira la nouvelle. Même pour un écrivain comme Flaubert, la formulation textuelle est le plus souvent seconde (mais certes pas secondaire) : «Le mot ne manque jamais quand on possède l'idée », écrit-il à George Sand25. À partir de là, le généticien peut comparer le monde stipulé par le scénario initial, dans lequel Félicité meurt à l'hôpital, et le monde mis en place à partir du scénario suivant, où Félicité meurt (dans sa chambre) pendant la Fête-Dieu.

Les changements du texte, qu'ils visent à produire des effets de sens ou des effets sonores (choix de rimes), ont le plus souvent des retombées sur le monde fictif projeté par ce texte 26 . Il est fréquent, on le sait, que le monde fictif soit manipulé pour faciliter la narration. Ainsi, Zola, planifiant le début du Rêve, prévoit de faire bouger son héroïne, chassée par la neige, pour pouvoir décrire plus commodément les sculptures du portail où elle s'est réfugiée : «Et alors, à mesure que la neige tombe et que le jour se lève, se servir de l'enfant qui change de place devant l'envahissement de la neige, pour décrire la porte ${ }^{27}$. »

À l'inverse, la logique propre au monde fictif («Si Félicien répare le vitrail, il faut un échafaudage $28 »)$ aura inévitablement des retombées sur le texte et sur les significations associées, au point qu'il est souvent impossible de déterminer quel niveau entraîne l'autre et difficile (mais nécessaire) de séparer les deux niveaux 29.

\section{Incomplétude ?}

En abordant les mondes fictifs, il faut dire quelques mots d'un problème qui a beaucoup agité les théoriciens de la fiction, celui de l'intégrité des mondes. Les mondes possibles de la logique sont des mondes virtuellement complets. Or les mondes de la fiction semblent incomplets, peuplés d'individus incomplets, c'est-à-dire ne possédant pas, comme c'est nécessairement le cas des objets réels, une valeur pour chacune des propriétés possibles : nous ne savons pas, et nous ne saurons jamais, combien d'enfants avait Lady Macbeth ou combien de fenêtres comporte la Maison Usher. On peut objecter que cette incomplétude

25. Lettre du 10 mars 1876.

26. Voir ci-dessus l'exemple élémentaire des « Sirènes ». Voir aussi dans « Le matériel et le virtuel... », art. cit., p. 26-27, un exemple tiré de Dostö̈evski montrant comment un changement de mode de narration entraîne par contrecoup un remaniement de la spatialité du monde fictionnel.

27. Plan du Rêve, $f^{\circ} 4$. On trouvera facilement d'autres exemples, ne serait-ce que les multiples cas où le besoin d'une nouvelle perspective narrative entraîne l'introduction d'un personnage surnuméraire, propre à orienter la focalisation.

28. Zola, plan du Rêve, f ${ }^{\circ} 71$.

29. Alors que les significations ne sont jamais totalement paraphrasables, tout ce qui relève de la référence se paraphrase d'ordinaire assez bien, sans reste ni ambiguïté (par exemple, on peut indiquer avec précision le même itinéraire en employant des formules différentes). Mais dans le domaine de la fiction, où c'est le texte qui, en produisant du sens, donne naissance au référent, il ne saurait pas y avoir de paraphrase exacte, même à ce niveau. 
n'est pas ontologique mais épistémique ${ }^{30}$ : nous ne connaissons pas ces nombres, mais ils existent ${ }^{31}$. Dans les mondes fictifs auxquels renvoient respectivement la pièce de Shakespeare et la nouvelle de Poe, ces propriétés sont censées être déterminées. Quoi qu'il en soit, dans le cadre de la genèse, l'indétermination des propriétés apparaît sous un jour différent.

Ce qui n'est pas déterminé dans le texte peut l'être dans le brouillon. Nous ne disposons pas de manuscrits des œuvres que nous venons de citer, mais on peut se reporter de nouveau à ceux de Madame Bovary. Les brouillons nous en disent, par exemple, beaucoup plus que la version finale sur la vie sexuelle d'Emma. Le roman, par choix esthétique de Flaubert ou par contrainte sociale incarnée par le procureur Pinard, laisse inexprimés ces détails scabreux. Mais même avec l'aide des manuscrits les plus explicites, nous ne pourrons jamais savoir combien d'orgasmes a eu Lady Bovary, car le monde projeté par le roman n'est pas le même que celui projeté par chacune des versions antérieures, et ce nombre n'est pas nécessairement identique dans chacun des mondes ${ }^{32}$. Il n'en reste pas moins que ce nombre est bien, dans chaque monde, un nombre déterminé, qui est parfois explicité, parfois non.

Mais il est plus instructif encore de se pencher sur la plus grande incomplétude de la plupart des versions avant-textuelles. Prenons d'abord le cas des mondes textuels. Quand Flaubert écrit, dans le troisième scénario d'Un cœur simple, cette phrase tronquée et trouée : «et quand elle exhala son dernier souffle... quand (une phrase très longue) cette vie terrestre s'éteignit... elle crut voir le Perroquet planant au-dessus de sa tête », il est clair que ce qu'il planifie, ce n'est pas une phrase incomplète, trouée, mais une longue et harmonieuse période pour conclure son conte. Ce qui est vrai sur le plan des mots l'est tout autant, et même davantage, sur le plan de l'univers représenté. Quand le scénario initial note simplement « elle meurt saintement », la description de la mort de Félicité apparaît évidemment incomplète par rapport à celle que nous présentera le texte définitif, mais elle est grosse de tous les détails qui surgiront ensuite (et de bien d'autres éventuellement). Il est clair que le monde fictif représenté n'a rien d'incomplet, c'est l'expression qui est laconique. Et on pourra dire paradoxalement la même chose de la plus développée, de la plus prolixe des versions.

30. C'est la conception que défend Marie-Laure Ryan. Doležel, Eco et Ronen ne sont pas du même avis, mais leurs arguments ne paraissent pas très convaincants. Cette manière de présenter les choses rappelle d'ailleurs celle de Kripke : « Un monde possible est donné par les conditions descriptives que nous lui associons. [...] Bien sûr, nous n'imaginons pas tout ce qui est vrai ou faux, seulement les choses qui ont rapport [avec notre hypothèse] ; en théorie, cependant, il faut décider de toutes choses pour donner une description complète du monde. Nous ne pouvons pas vraiment imaginer cela, sinon en partie ; et c'est cela, un "monde possible" ", La Logique des noms propres, op. cit., p. 32. Et aussi : «Bien entendu, quand nous spécifions une situation contrefactuelle, nous ne décrivons pas le monde possible tout entier, mais seulement la portion qui nous intéresse » (p. 37, n. 16). Nous avons vu que les situations contrefactuelles pouvaient être assimilées à des micro-romans.

31. Pour s'en persuader, il suffit de considérer le trouble qui saisit la fiction quand un écrivain fait mine de rabattre l'incomplétude de la représentation textuelle sur le monde représenté (Sterne, Pirandello, Stoppard...). Pour l'analyse de la genèse d'un exemple particulièrement déstabilisant de ce procédé chez Joyce, voir D. Ferrer, « Peut-on parler de métalepse génétique ?», dans J. Pier et J.-M. Schaeffer (dir.), Métalepses : Entorses au pacte de représentation, Éditions de l'École des hautes études en sciences sociales, 2005. En ligne sur <www.item.ens.fr/index. php?id=13987>.

32. Voir le cas emblématique de Gervaise qui n'a pas le même nombre d'enfants dans le monde fictif de L'Assommoir et dans celui de La Bête humaine. 
Une mise en garde toutefois. Nous avons l'habitude de combler les lacunes de notre connaissance du monde fictif en y important massivement nos connaissances extra-fictionnelles. Flaubert ne précise pas dans Un coeur simple le nombre de jambes de ses personnages ni la longitude de Rouen, mais nous tenons pour acquis que Félicité a deux jambes et que la ville qu'elle habite est située à l'ouest de Paris ${ }^{33}$. Sur le modèle de cette inférence légitime, nous avons tendance à combler les trous des versions elliptiques rencontrées dans les manuscrits au moyen d'emprunts à la version définitive. C'est une commodité pour nos imaginations défaillantes, mais il faut être conscient que rien ne nous y autorise en droit. En matière de genèse, on ne peut pas raisonner toutes choses étant égales par ailleurs.

\section{Possibles}

Si l'on admet l'idée que mondes textuels et mondes fictifs se superposent, parfois inextricablement ${ }^{34}$, dans l'avant-texte, il faut préciser leur statut. C'est la complexité de ce statut qui fait la particularité des études génétiques. Nous l'avons vu, ces mondes sont à la fois actuels et virtuels. Ils sont indéniablement présents à travers les documents, la preuve en est qu'un lecteur peut en prendre connaissance, lire un brouillon à cette fin comme on lirait un texte définitif, accéder à un univers fictif à partir d'un scénario presque aussi bien qu'à travers un roman achevé. Pour l'écrivain lui-même, les mondes qu'il crée ont une existence dans l'instant de leur inscription. Comme le dit Jean Bellemin-Noël, ce qui est écrit

n'est jamais à l'avance et par destination conçu comme un déchet; et même le «premier jet », s'il est voué à se trouver amendé, a des chances de rester en l'état. C'est toujours après coup qu'on reconnaît un fourvoiement, une impasse, un échec. L'inabouti a d'abord été, fût-ce l'espace d'un instant, du provisoirement abouti 35 .

Ce provisoirement définitif restera toutefois définitivement marqué du sceau du provisoire, en suspens, ayant des chances d'aboutir jusqu'au moment de l'achèvement. Son existence est projective, explicitement ou implicitement modalisée.

Il n'est pas rare que la modalisation s'exprime directement. Par exemple dans cette note de Stendhal en marge de Lucien Leuwen : «Faut-il placer ce deuxième entretien avant le second duel? Ce serait plus narratif. » Parfois, ce qui semble posé est aussitôt mis en suspens : « Il fera croire à Leuwen qu'elle est accouchée en secret. Du Poirier ne dit pas cela aux vicomtes mais il le pense, et moi auteur je me dis : Est-ce bon ${ }^{36}$ ? » Dans les Ébauches et Plans de Zola, qui ont souvent l'avantage de présenter à découvert ce qui ailleurs reste implicite, les formes du conditionnel sont très nombreuses, portant tantôt sur les mondes fictionnels et tantôt sur les mondes textuels :

33. C'est ce que Marie-Laure Ryan appelle le principe de «minimal departure ». Sauf spécification contraire et jusqu'à plus ample informé, nous supposons que le monde fictif est identique en tout point à celui que nous considérons comme le monde réel.

34. Dans la mesure où toute phrase de l'avant-texte peut être lue comme relevant d'une attitude propositionnelle (dans le plan du Rêve, la proposition « la neige tombe » équivaut à « Je [Zola] décide (ou j'envisage) que la neige tombera au début du roman »), la distinction monde textuel/monde fictionnel pourrait être ramenée à une distinction de relde dicto dont il serait impossible, dans ce contexte bien particulier, de lever l'ambiguïté.

35. «Reproduire le manuscrit, présenter les brouillons, établir un avant-texte », Littérature, n ${ }^{28}$, 1977, p. 7.

36. Stendhal, Euvres romanesques complètes, édition établie par Yves Ansel, Philippe Berthier et Xavier Bourdenet, Paris, Gallimard, 2007, p. 923. 
Si je reprends l'église, certaines modifications importantes en résultent. D'abord mon Hautecœur redevient évêque. Mais je voudrais lui conserver le blason et son histoire. Peut-être le pourrai-je en lui donnant une chapelle dans la cathédrale. Les Hautecœur seraient une vieille famille du pays. Un château ruiné dans les environs, et toutes les légendes que je voudrais, les mortes jeunes, dans le bonheur. (Leur corps est là dans la chapelle Cette chapelle dédiée à Saint George aurait donc le vitrail du saint, une montée en haut de l'ogive, dans la rose, des armes des Hautecœur. Angélique verrait donc continuellement ce vitrail de Saint George et les armoiries des Hautecœur. De plus, on lui aurait raconté l'histoire de la famille. Et tout ça prendrait place au chapitre IV, avant l'arrivée du prince Charmant, grâce à la fenêtre éclairée 37 .

Dans la plupart des manuscrits, toutefois, la modalisation est sous-entendue. Si la fiction repose bien sur un énallage de modalité (indicatif pour conditionnel) - il faudrait dire que les avant-textes des fictions sont composés d'énallages de modalité à multiple détente (indicatif assertif, mis à la fois pour un conditionnel marquant une suspension projective et pour un impératif prescrivant une réalisation future, annonçant un indicatif narratif mis pour un conditionnel marquant la suspension fictive...).

Quand Flaubert écrit « une attaque à l'hôpital / elle meurt saintement », il indique quelque chose comme « elle pourrait mourir saintement d'une attaque à l'hôpital », ou bien « il faudrait qu'elle meure saintement... ». De même, quand il écrit les mots « un gigantesque perroquet », cela ne signifie pas que Flaubert croit, au moment où il les écrit, que les mots « gigantesque perroquet » figureront tels quels dans l'œuvre définitive. On sent bien qu'il est ici à la recherche d'un rythme anapestique qui reste à perfectionner. Les écrivains procèdent par essai et erreur, posent des pierres d'attente. Il s'agit bien d'une prescription modalisée. Comme le dit cette phrase de Zola, d'application quasi universelle dans l'avant-texte : « Pourtant, cela est à régler, lorsque j'aurai tous les éléments ${ }^{38}$. »

Les mondes avant-textuels sont donc des mondes possibles. Mais par rapport à quoi sontils possibles ? Quel est le point d'ancrage, le monde actuel par rapport auquel les situer ? Tout dépend de la perspective adoptée.

D'un point de vue rétrospectif, le monde actuel c'est l'œuvre achevée, telle qu'en ellemême l'éternité la change. Les mondes possibles, accessibles depuis ce monde actuel, ce sont ceux que le généticien range dans l'avant-texte de cette œuvre particulière. Les règles

37. Plan du Rêve, fos 52-53, transcription simplifiée. Voir le très utile site que l'équipe «Zola » de l'ITEM et la BnF ont consacré au Rêve <http://gallica.bnf.fr/html/dossiers/Zola/>.

38. Ébauche de La Bête humaine, $\mathrm{f}^{\circ} 593$. 
d'accessibilité sont implicitement définies par le geste de partage qui définit l'avant-texte. Elles peuvent donc varier quelque peu d'un critique à l'autre 39 .

Du point de vue de l'écriture en cours, chacun des mondes provisoirement adoptés, fûtce, comme le dit Bellemin-Noël, «l'espace d'un instant », au détour d'une phrase, représente tour à tour le monde actuel. Comme chez les « réalistes modaux », l'actualité est une question de point de vue. Les mondes accessibles à partir de ce monde-là sont les potentialités qui s'ouvrent à partir de ce point ${ }^{40}$. Zola encore : " L'important est de savoir si j'enfermerai le roman dans le monde des filles. Ou si j'aurai un autre monde à côté. Ce monde à côté pourrait me servir à dramatiser un peu l'action ${ }^{41}$. »

Ces virtualités logiques, qui ne cessent de se présenter jusqu'au dernier moment, même chez un écrivain réputé déterministe (« je ne vais pas jusqu'à dire qu'il n'y a qu'un dénouement possible pour chaque roman; il y a plusieurs dénouements logiques, selon que le romancier dispose les documents dont il reste le maître, tant qu'il ne les a pas employés 42 »), s'incarnent dans le foisonnement des variantes, versions, ratures, alternatives non résolues. Bien entendu, d'autres éventualités se sont présentées, qu'aucune trace n'atteste, mais c'est à partir de ces possibles attestés que doit travailler la critique génétique.

\section{Nécessaire ?}

Après avoir évoqué cet hypothétique «monde à côté » qui pourrait lui servir à dramatiser l'action de Nana, Zola ajoute : « Je crois que ce bout d'intrigue est nécessaire. » En l'espace d'un instant et comme sans y penser, il est passé de la virtualité à la nécessité.

Il n'est pas facile de définir ce que peut être le nécessaire dans un cadre génétique. Il ne faut pas le confondre avec l'évidence artistique qui s'impose dans l'œuvre achevée, parvenant à la « soustraire aux contingences du temps » en l'enfermant dans « les anneaux nécessaires d'un beau style 43 ». On pourra s'inspirer de la conception d'Eco pour qui, dans les mondes

39. « Définir l'avant-texte comme un objet construit, c'est admettre une pluralité de constructions possibles » (Louis Hay, «"Le texte n'existe pas”. Réflexions sur la critique génétique », Poétique, n 62, 1985, p. 152). «L'avant-texte est le produit [d'une] lecture nécessairement spécialisée qui implique l'adaptation d'une méthode d'analyse textuelle aux réalités mouvantes de la genèse. Pour un même dossier de genèse, il pourra donc y avoir autant d'avant-textes que de points de vue choisis pour l'interpréter » (Pierre-Marc de Biasi, «L'analyse des manuscrits et la genèse de l'œuvre », Encyclopaedia Universalis, vol. Symposium, Paris, Bordas, 1985).

40. Il y a évidemment une certaine ressemblance (et même une certaine symétrie) entre ces virtualités et les anticipations qui se présentent à l'esprit du lecteur au fur et à mesure de sa lecture de l'œuvre achevée, les «promenades inférentielles » chères à Eco (Lector in fabula, op. cit., p. 154-159). Mais, à la différence des projections de l'écrivain, révélées par les manuscrits, les anticipations du lecteur ne sont nulle part attestées, elles sont elles-mêmes l'objet d'une inférence de la part du critique. Ce qui est, en revanche, occasionnellement attesté, c'est l'anticipation par l'écrivain des anticipations du lecteur.

41. Ébauche de Nana. Quand Zola parle de «mondes », ici ou dans la citation placée en exergue du présent article, il n'est pas douteux qu'il se réfère d'abord aux milieux sociaux (aux sphères socioprofessionnelles dans lesquels évoluent ses personnages), mais cet usage (comme celui du mot sphère) repose bien sur une image, plus ou moins usée par la lexicalisation, d'univers parallèles et relativement étanches.

42. Lettre du 26 novembre 1879, citée par Colette Becker dans « Cela "s'établira en écrivant" (Zola) », préface à : Émile Zola, La Fabrique des Rougon-Macquart. Édition des dossiers préparatoires, publiés par Colette Becker, avec la collaboration de Véronique Lavielle, vol. III, Paris, Honoré Champion, 2006.

43. Marcel Proust, À la recherche du temps perdu, III, Paris, Gallimard, coll. « Bibliothèque de la Pléiade », 1954, p. 889 . 
fictionnels (et plus généralement dans les mondes culturellement construits), la nécessité se présente sous deux aspects, une nécessité « topico-sensible » (déterminée au départ par le choix d'un champ de pertinence) et une nécessité structurale (qui consisterait en une interdépendance fonctionnelle des propriétés : Othello et Desdémone sont réciproquement et nécessairement définis comme « mari de Desdémone » et « épouse d'Othello »)44. La nécessité structurale est celle qui règne à l'intérieur des mondes leibniziens. Dans l'hypothèse où Tarquin devient roi, il est nécessaire qu'il soit criminel et qu'il finisse exilé de Rome : il est libre d'éviter le crime et l'exil, mais seulement s'il choisit une destinée au cours de laquelle il ne monte pas sur le trône. Le dispositif des mondes juxtaposés permet à Athéna de démontrer que tout changement local met en cause la totalité. Depuis la perspective surplombante qui est la sienne, la génétique devrait pouvoir mettre en évidence une interdépendance comparable.

Il faut partir de la notion de «variante liée », définie par Almuth Grésillon comme :

[...] toute variante qui intervient sous la pression d'une donnée contextuelle (intra- ou inter-énoncé) ou textuelle. Cette pression, dont les différents aspects restent à préciser, a pour conséquence la nécessité de procéder au remplacement de X. Autrement dit, sous l'effet d'une donnée A, l'élément $\mathrm{X}$ est exclu45.

Il va de soi que raturer un «nous » et le remplacer par un « je » implique qu'on accorde le verbe suivant à la première personne du singulier. Le changement de terminaison est donc bien une variante liée. Il est tout aussi clair que «Salomon », qui était le prénom du père de Mina, l'héroïne de Rose et vert, a été remplacé par «Pierre » en liaison avec la substitution de «nous avons beau avoir des millions, nous ne serons jamais que la femme et la fille d'un marchand» à « J'aurais beau avoir des millions, disait Mina à sa mère, en Allemagne dans le pays de la noblesse de race je ne serais jamais qu'une juive c'est à dire un être souverainement envié et malgré cette envie presque toujours méprisé », c'est-à-dire en relation avec le changement de statut de Mina, qui devient, comme Stendhal a eu l'obligeance de le noter en travers de son manuscrit, « non juive 46 ». Mais il faut aller plus loin et généraliser cette notion de liaison, tout en la modulant 47 . On est amené à constater qu'aucune variante n'est véritablement libre et que toute modification vient nécessairement s'inscrire dans un ou plusieurs systèmes qui exercent sur elle des contraintes de divers ordres. Les généticiens, qui ne disposent pas, pour les éclairer, du secours d'Athéna, ne sont pas toujours à même d'expliciter ces contraintes

44. Lector in fabula, op. cit., chap. VIII. Ronen (Possible Worlds in Literary Theory, op. cit., p. 54) souligne à quel point cette conception de la nécessité diffère de celle de la logique modale.

45. Almuth Grésillon, «Les variantes de manuscrits : critères et degrés de pertinence », dans La Publication de manuscrits inédits, éd. Louis Hay et Winfried Woesler, Berne, Peter Lang Verlag, 1979, p. 183. Dans «Linguistique et génétique des textes : un décalogue » (Le Français moderne, numéro spécial : «Tendances actuelles de la linguistique française », Paris, CILF, 2008), Almuth Grésillon et Jean-Louis Lebrave adoptent une définition plus restrictive : «La variante sera dite liée quand elle est due à des contraintes de langue (morphologiques, lexicales, syntaxiques ou de règles d'enchainement textuel) ou si elle n'est que l'effet grammaticalement nécessaire d'une variante première. Les variantes non liées sont dites libres. » On comprend qu'une telle définition soit plus opératoire pour l'analyse linguistique, mais elle est sans doute moins productive d'un point de vue théorique. Dans son article de 1979, Almuth Grésillon refusait de considérer qu'une variante puisse être libre (p. 186).

46. Au folio 6 du brouillon intitulé « Tamira Wangen».

47. Almuth Grésillon parle de «restriction des latitudes [...] plus ou moins forte » («Les variantes de manuscrits », art. cit., p. 183). 
quand elles n'ont pas la belle simplicité des lois de la grammaire. Aux échecs, il est facile d'énoncer les règles qui autorisent ou interdisent le déplacement d'une pièce ; il est beaucoup plus difficile d'analyser les rapports qui, dans une position donnée, relient cette pièce à toutes les autres et qui contraignent son action. C'est que ces rapports sont dynamiques, redistribués à chaque mouvement et évoluant en fonction des projets des joueurs. Il en va de même au cours de la genèse.

S'il est vrai que, dans la pièce de Shakespeare, être «mari de Desdémone » et «épouse d'Othello » sont des propriétés nécessaires d'Othello et de Desdémone, ce n'était pas forcément le cas dans les manuscrits : pour peu que Shakespeare ait décidé que son topic n'était pas la jalousie, Othello aurait pu être célibataire. Les deux formes de nécessité sont étroitement imbriquées : la nécessité structurale ne vaut qu'en fonction du choix des topics, or ceux-ci sont constamment adaptés et réinterprétés. Après tout, même l'accord du verbe à la première personne du singulier après un « je », que nous avions pris comme exemple de variante liée, cesse de s'imposer si une construction délibérément a-grammaticale vient à l'ordre du jour, ou encore si la locution est attribuée à un paysan normand.

Pour reprendre l'exemple fameux du poème Liberté, on sait qu'un basculement de topic s'est littéralement imposé en cours d'écriture, une soudaine mutation, ou plutôt une réinterprétation du champ de pertinence (« je me suis vite aperçu que le seul mot que j'avais en tête était le mot liberté48 »), qui s'est traduite superficiellement par une unique variante, mais qui a bouleversé l'ensemble des relations internes au poème. C'est ce qui a permis à Louis Hay d'avancer que le texte apparaît, à la lumière de sa genèse, comme un «possible nécessaire 49 ».

\section{Accessibilités}

On comprend que le problème crucial est celui du passage d'un monde à l'autre. Nous avons vu que la logique modale définissait des relations entre les mondes, dites relations d'accessibilité. Elles déterminent à quel monde on peut accéder à partir de tel autre. L'accessibilité peut être transitive ou non transitive (si un monde A est accessible à un monde $\mathrm{B}$, qui lui-même est accessible à un monde $\mathrm{C}$, A n'est pas forcément accessible à $\mathrm{C}$ ), symétrique ou non symétrique (si un monde $\mathrm{A}$ est accessible à un monde $\mathrm{B}$, B n'est pas toujours accessible à $\mathrm{A}$ ), réflexive ou non réflexive (c'est-à-dire qu'un monde n'est pas nécessairement accessible à lui-même).

Comment définir de telles relations dans le cadre de la genèse ? Il serait passionnant (et peut-être utopique) de pouvoir déterminer rigoureusement ce qui fait qu'on peut ou qu'on ne peut pas passer d'un état $\mathrm{X}$ à un état $\mathrm{Y}$, ce qui fait qu'une variante est possible ou impossible à tel moment, en tel point de l'avant-texte.

Si l'on parvenait à définir une telle relation d'accessibilité, on pressent qu'elle combinerait des asymétries partielles et croisées. Dans le sens rétrograde, un état X est accessible sans

48. Paul Éluard, « Poésie de circonstance », cité par Louis Hay dans La Littérature des écrivains. Questions de critique génétique, Paris, José Corti, 2002, p. 57.

49. «"Le texte n'existe pas". Réflexions sur la critique génétique », art. cit., p. 158. Voir aussi cette formulation : « La genèse du texte offre à la critique [...] un critère d'intelligibilité en montrant ce qu'une œuvre aurait pu être et ce qu'elle est. Ainsi, le jugement esthétique peut induire la nécessité du texte à partir des possibles qu'il a expérimentés » (La Littérature des écrivains, op. cit., p. 29). 
difficulté à partir de l'état suivant $\mathrm{Y}$, alors que l'accession de $\mathrm{Y}$ à partir de $\mathrm{X}$ ne va pas de soi. Bergson utilise précisément l'exemple de l'œuvre d'art pour nous mettre en garde contre les mirages du possible :

Quand un musicien compose une symphonie, son œuvre était-elle possible avant d'être réelle ? Oui, si l'on entend par là qu'il n'y avait pas d'obstacle insurmontable à sa réalisation. Mais de ce sens tout négatif du mot on passe, sans y prendre garde, à un sens positif : on se figure que toute chose qui se produit aurait pu être aperçue d'avance par quelque esprit suffisamment informé, et qu'elle préexistait ainsi, sous forme d'idée, à sa réalisation ; - conception absurde dans le cas d'une œuvre d'art, car dès que le musicien a l'idée précise et complète de la symphonie qu'il fera, sa symphonie est faite. Ni dans la pensée de l'artiste, ni, à plus forte raison, dans aucune autre pensée comparable à la nôtre, fût-elle impersonnelle, fût-elle même simplement virtuelle, la symphonie ne résidait en qualité de possible avant d'être réelle ${ }^{50}$.

Pour expliquer plus clairement la notion d'accessibilité asymétrique, on reprend souvent l'exemple suivant : à partir du monde contemporain, où le téléphone existe, un monde sans téléphone est facilement concevable, mais à partir d'un monde sans téléphone on ne peut pas concevoir un monde où le téléphone existerait ${ }^{51}$. Toutefois, comme le fait remarquer Eco ${ }^{52}$, cette présentation oublie, ou fait mine d'oublier, qu'il a existé un Graham Bell qui, dans un monde sans téléphone, a su concevoir (et faire advenir) un monde où le téléphone existe. La symphonie dont parle Bergson, qui n'était pas possible, au sens fort, s'écrit cependant. Dans le domaine de la création artistique, c'est bien d'invention qu'il s'agit et les inventions sont des transgressions par rapport aux conventions (génériques ou autres) qui prescrivent les possibilités ouvertes. Les grands innovateurs sont tout à fait comparables à Graham Bell. Nous avions tout à l'heure choisi avec confiance la proposition « toute œuvre a un commencement» comme exemple de la relation de nécessité, puisque cette proposition paraît devoir être vraie dans tous les mondes accessibles à partir du monde actuel. Or, grâce à sa forme circulaire, Finnegans Wake n'a ni commencement ni fin. De même, jusqu'à Joyce, seuls étaient possibles des œuvres littéraires écrites dans un langage naturel, ce qui n'a pas empêché l'écriture de Finnegans Wake. Toutefois l'abandon de la contrainte des lexiques existants ne signifiait pas que tout mot devenait possible à tout instant. De nouvelles règles d'accessibilité se sont, progressivement, mises en place : telle est l'essence du «Work in Progress » joycien.

On pourrait aller plus loin et dire que, dans une écriture quelle qu'elle soit (car la symphonie dont parle Bergson n'est pas nécessairement révolutionnaire), tout état $\mathrm{Y}$ représente une transgression, aussi minime soit-elle, par rapport au cadre qui a présidé à l'élaboration de l'état X précédent : il n'est pas complètement pensable à partir de ce cadre, il est inaccessible à partir de $\mathrm{X}$.

En un autre sens, $\mathrm{X}$ est par ailleurs inaccessible à partir de $\mathrm{Y}$, car le projet originel de $\mathrm{X}$ est subtilement (ou radicalement) modifié par le changement qui aboutit à $Y$, de telle manière que la perspective originelle est irrécouvrable : c'est la raison pour laquelle les créateurs sont aussi peu

50. La Pensée et le mouvant, Paris, Alcan, 1934, p. 20.

51. Cet exemple (qui a l'inconvénient de psychologiser la notion d'accessibilité) provient du célèbre manuel de G.E. Hughes et M. J. Cresswell, An Introduction to Modal Logic, London, Methuen, 1972, p. 77-78.

52. Lector in fabula, op. cit., p. 174. 
dignes de foi quand ils parlent de la genèse de leur œuvre. Parlant du point de vue de l'œuvre achevée, le projet de départ, archaïque, frappé d'obsolescence, leur est devenu inaccessible.

Pour les mêmes raisons, l'accessibilité génétique est non transitive : depuis X, il n'est pas possible d'avoir accès aux mondes $\mathrm{Za}, \mathrm{Zb}, \mathrm{Zc}$... qui seront accessibles depuis $\mathrm{Y}$, et moins encore aux mondes qui seront accessibles à partir de l'un des mondes Z.

Un tel dispositif devrait permettre de prendre en compte les virtualités du processus de création sans avoir à nier sa dimension temporelle comme c'était le cas dans le système de Leibniz. Par l'intermédiaire d'une métaphore spatiale (l'accessibilité entre les mondes) on pourra essayer d'analyser la temporalité non linéaire, discontinue, accidentée, qui est celle de la genèse.

Tout ceci reste à approfondir et à systématiser, mais il n'est pas certain que nous parvenions jamais à définir strictement les règles génétiques de l'accessibilité dans le cadre de la logique aléthique (celle qui se fonde sur les catégories du possible, du nécessaire, du contingent, ou de l'impossible). Peut-être ces catégories ne sont-elles pas les plus appropriées à la genèse ? Peut-être faudra-t-il faire appel aux autres sortes de logique modale, qui conservent une structure semblable mais donnent un autre sens aux opérateurs de possibilité et de nécessité de manière à refléter les catégories déontiques (permis, interdit, obligatoire) ou axiologiques (bien, mal, neutre, quel que soit le contenu qu'on met sous ces critères), voire celles des logiques temporelles (dorénavant, quelquefois, jamais) linéaires ou arborescentes ? Peut-être tout cela se révélera-t-il trop statique et faudra-t-il se référer aux logiques dynamiques, ou aux logiques floues qui tentent de se mettre en place actuellement ?

Avant de chercher plus loin, il semble toutefois de bonne méthode d'explorer ce que le cadre aléthique de base pourrait nous apporter, moyennant certaines adaptations. Ou plus exactement, ce sont les indispensables adaptations qui nous permettront de mieux comprendre la spécificité de notre objet.

Monde(s)

On voit que le recours à la notion de mondes possibles ouvre un certain nombre de perspectives. Ce n'est qu'à l'usage que nous pourrons savoir si une telle manière de poser les problèmes se révélera féconde, si elle affectera notre manière de considérer notre objet et si elle pourra infléchir, à la marge ou en profondeur, notre pratique quotidienne.

Pour qui ne serait pas convaincu, il n'est pas indispensable d'adopter en bloc l'ensemble du dispositif. La notion se laisse décomposer. La génétique pourrait laisser de côté la sémantique des mondes possibles et (peut-être ?) envisager de se passer de la notion de mondes fictionnels, jugée contradictoire par certains, mais pas de celle de monde, c'est-à-dire, en somme, de la dimension extensionnelle de la genèse, que nos travaux ne devraient pas négliger, ni surtout de celle de mondes, au pluriel ${ }^{53}$, comme corrélat de celle de fabrication de monde, «worldmaking » pour reprendre le titre d'un ouvrage de Nelson Goodman ${ }^{54}$. Pour ce dernier, comme nous l'avons indiqué plus haut, il n'est pas de monde

53. Qu'on accepte ou non la conception du possible défendue par Leibniz, son apologue introduit l'idée d'une pluralité d'univers représentés, à la fois séparés et mathématiquement coordonnés.

54. Ways of Worldmaking, traduit par Manières de faire des mondes (Nîmes, Jacqueline Chambon impr., trad. MarieDominique Popelard, 1992). 
donné, mais seulement des mondes construits 55 , y compris celui que nous tenons pour la réalité. Un tel constructivisme radical n'est pas pour déplaire à la critique génétique, d'autant qu'on pourrait dire qu'il est en fait un re-constuctivisme ou un révisionnisme radical («Pour nous, la construction d'un monde se fait toujours à partir de mondes qui sont déjà à disposition ; faire c'est refaire $56 »)$. On retrouve d'ailleurs, passés en revue dans Manières de faire des mondes, les mécanismes génétiques familiers : composition et décomposition, mise en valeur sélective, réorganisation, suppression et ajout, déformation.

Quant au programme liminaire de cet ouvrage (« Sans avoir la prétention d'en remontrer aux dieux ou autres faiseurs de monde, [...] je veux illustrer et commenter quelques-uns des procédés qui entrent dans la fabrication des mondes 57 »), les généticiens pourraient le faire leur, qu'il s'agisse de la fabrication des mondes restreints rencontrés dans les manuscrits ou de la construction effective des vastes mondes de type goodmaniens ${ }^{58}$. Forts de leur connaissance approfondie des mécanismes de genèse, ils ont certainement leur mot à dire sur le sujet.

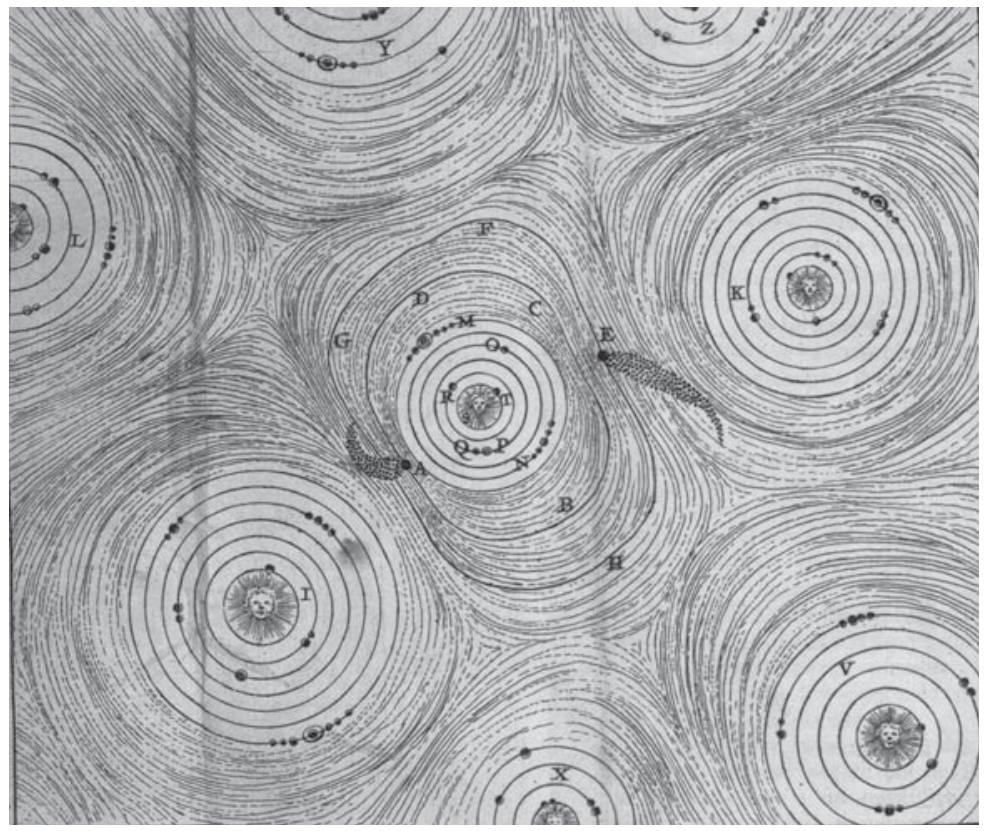

Nicolas Bion.

L'usage des globes célestes et terrestres et des sphères suivant les différents systèmes du monde (1699)

55. Voir notamment Of Mind and Other Matters, Cambridge, Harvard University Press, 1984, p. 125. Malgré la différence de force illocutoire entre les verbes « construire » et « stipuler », il ne semble pas pertinent, du point de vue génétique, de maintenir une distinction entre les mondes construits de Goodman et les mondes stipulés de Kripke. La stipulation d'un monde suppose évidemment une construction, fût-elle embryonnaire. Mais la construction de mondes (activité dans laquelle nous sommes perpétuellement engagée, que nous en ayons conscience ou non) ne suppose-t-elle pas une stipulation implicite (soit un monde tel que...) ? C'est en tout cas ainsi qu'on est obligé d'analyser la production d'une œuvre.

56. Manières de faire des mondes, op. cit., p. 15 (traduction modifiée).

57. Ibid., p. 15 (traduction modifiée).

58. C'est-à-dire ce qu'on appelle ordinairement les « visions du monde », appellation trompeuse selon Goodman, car elle suggère l'existence d'un monde préalable aux visions et séparable d'elles. 


\section{Appendice terminologique : Diégèse/monde(s) - récit/fiction}

Une question de vocabulaire mérite sans doute d'être soulevée. Pourquoi ne pas utiliser le terme narratologiquement établi de diégèse ? Si les « livres des destinées » de Leibniz sont des récits et ses « destinées » des histoires, ses «mondes » semblent correspondre assez bien à ce que Genette, après Souriau, appelle diégèse $e^{59}$. Il faut d'abord remarquer que cette notion est en fait relativement peu utilisée dans la narratologie classique, peut-être parce qu'elle est brouillée par la proximité verbale de la diegesis aristotélicienne, mais surtout parce qu'elle est structurellement marginale par rapport au couple histoire/récit (comme le référent reste marginal dans la linguistique saussurienne par rapport au couple signifié/signifiant) et parfois confondue avec l'histoire60.

L'acception proposée par l'index terminologique de Figure II ( « l'univers spatio-temporel désigné par le récit ») comporte une importante nuance par rapport à la définition de Souriau ( « tout ce qui est censé se passer, selon la fiction que présente le film; tout ce que cette fiction impliquerait si on la supposait vraie », p. 240). Comme le remarque bien Genette dans sa préface à la Logique des genres littéraires de Käte Hamburger : " on ne peut étudier le récit de fiction à la fois comme récit et comme fiction : le "comme récit" de la narratologie implique par définition que l'on feigne d'accepter l'existence [...], "avant" le récit, d'une histoire à raconter ; le "comme fiction" de Käte Hamburger implique au contraire que l'on refuse cette hypothèse 61 ». Le Genette de Figure II considère la diégèse à partir du récit ${ }^{62}$, qui la désigne à travers l'histoire, comme un signifiant désigne le référent à travers son signifié (sans qu'il y ait lieu de se demander si cette histoire et cette diégèse préexistent au récit ou sont engendrées par lui). En revanche, la diégèse de Souriau est inséparable de la fictionnalité à laquelle elle est liée par un rapport d'inférence (« tout ce qui est censé se passer, selon la fiction [...] tout ce que cette fiction impliquerait »). Elle est l'objet d'une modalisation forte (« selon la fiction [...] si on la supposait vraie »).

L'idée de modalisation et celle d'inférences pratiquées à partir d'entités fictionnelles sont capitales pour une théorie de la fiction et plus encore pour une théorie génétique, mais on voit combien le terme de diégèse est ambigu à cet égard. Il a donc semblé préférable d'y renoncer dans le présent article et de se servir de celui de « monde », sans doute plus ambigu encore mais universellement compris.

En dehors de la tradition philosophique et logique63, monde est couramment utilisé dans bien d'autres contextes, et notamment par la critique littéraire. On parle, de manière

59. Eugène Souriau, « La structure de l'univers filmique et le vocabulaire de la filmologie », Revue internationale de filmologie, $\mathrm{n}^{\circ} 7-8,1951$, p. 231-240.

60. Genette reconnaît que sa formulation ambiguë de «Discours du récit » (dans Figures III, Paris, Éditions du Seuil, 1972) a sa part de responsabilité dans cette confusion. Il s'est efforcé de la dissiper dans Palimpsestes (Paris, Éditions du Seuil, 1982), où il distingue nettement l'histoire, « succession d'événements et/ou d'actions » et la diégèse, « univers où advient cette histoire » (p. 342) et encore dans Les Nouveaux Discours du récit, Paris, Éditions du Seuil, 1983.

61. Paris, Éditions du Seuil, 1986, p. 13.

62. Il faut entendre « récit » au sens large : les passages descriptifs ont, à l'évidence une fonction de désignation de l'univers spatio-temporel égale et même supérieure à celle des passages purement narratifs. Remarquons que dans le domaine filmique où se place Souriau la distinction récit/description n'est pas forcément pertinente.

63. Saul Kripke affirme que l'appellation de «mondes possibles » résulte d'un « accident terminologique » et aurait aussi bien pu être remplacée par «états possibles du monde » ou « situations contrefactuelles », ou encore « histoires (histories) possibles du monde » (La Logique des noms propres, op. cit., p. 171). 
un peu vague, du «monde de Balzac » (ou de celui de Verlaine ou de Shakespeare). Par ailleurs, des théoriciens comme Ingarden ou Lotman ont utilisé le mot, dans une optique phénoménologique ou sémiologique, pour désigner un principe de cohérence interne 64 . J'espère avoir montré que la prise en compte d'une pluralité de mondes alternatifs pouvait ouvrir des perspectives différentes.

64. Voir à ce propos l'analyse de Ruth Ronen, Possible Worlds in Literary Theory, op. cit., p. 95-107. 
DANiel FerRer est directeur de recherche à l'ITEM (CNRS-ENS). Il a publié notamment Poststructuralist Joyce (avec Derek Attridge, Cambridge University Press, 1984), Virginia Woolf and the Madness of Language (Routledge, 1990), L'Écriture et ses doubles : genèse et variation textuelle (avec Jean-Louis Lebrave, CNRS Éditions, 1991), Ulysse à l'article : Joyce aux marges du roman (avec Claude Jacquet et André Topia, Du Lérot, 1992), Genèses du roman contemporain : Incipit et entrée en écriture (avec Bernhild Boie, CNRS Éditions, 1993), Writing its own wrunes for ever: Essays in Joycean Genetics (avec Claude Jacquet, Du Lérot, 1998), Pourquoi la critique génétique? Méthodes, théories (avec Michel Contat, CNRS Éditions, 1998), Bibliothèques d'écrivains (avec Paolo D’Iorio, CNRS Éditions, 2001), Genetic Criticism: Texts and Avant-textes (avec Jed Deppman et Michael Groden, Philadelphia, Pennsylvania University Press, 2004), La Textologie russe (avec Andrei Mikhailov, CNRS Éditions, 2007). Avec Vincent Deane et Geert Lernout, il édite les Finnegans Wake Notebooks at Buffalo (Brepols, 12 volumes publiés, 48 à paraître).

Daniel Ferrer, daniel.ferrer@ens.fr

\section{Mondes possibles, mondes fictionnels, mondes construits et processus de génèse}

La critique génétique ne peut pas ignorer la dimension extensionnelle de la genèse. Elle aurait avantage à s'appuyer sur l'idée de monde, sans doute plus utile, à cet égard, que la notion de diégèse, parce qu'elle permet de concevoir une pluralité de mondes stipulés. En prenant soin de distinguer mondes textuels et mondes fictionnels, on pourra s'inspirer de la logique des mondes possibles pour essayer de préciser le statut des mondes, à la fois réels et virtuels, créés au cours de la genèse et pour définir les règles d'accessibilité qui régissent les rapports entre ces mondes.

Genetic criticism cannot overlook the extensional dimension of genesis. It would be profitable to draw on the idea of world. In this regard, it would be more useful than the notion of diegesis, because it enables us to conceive of a plurality of stipulated worlds. By carefully distinguishing between textual worlds and fictional worlds, we can take example on the logic of possible worlds to try to specify the status of the worlds, both real and virtual, that are created during the genesis, and define the accessibility rules regulating the relations between these worlds.

Die „critique génétique“ kann das Wissen um die erweiterte Dimension der Genese nicht ausblenden. Sie wäre gut beraten, wenn sie in diesem Zusammenhang mit der Vorstellung von einer Welt arbeiten würde, weil diese, besser als der Begriff der Diegese, es ermöglicht, eine Pluralität der Welten anzunehmen. Vorausgesetzt, man unterscheidet sorgfältig zwischen den Welten des Textes und den Welten der Fiktion, könnte man in Anlehnung an die Logik der möglichen Welten versuchen, den Status der im Laufe des Schaffensprozesses entstehenden (sowohl realen wie virtuellen) Welten zu präzisieren und die Regeln zu begreifen, nach denen sich diese Welten zueinander verhalten.
La crítica genética no puede ignorar la dimensión extensional de la génesis. Sería provechoso para ella basarse en la idea de mundo, sin duda más útil, en este sentido, que la noción de diégesis, porque permite concebir una pluralidad de mundos estipulados. Tomando cuidado en distinguir mundos textuales y mundos ficcionales, es dado inspirarse en la lógica de los mundos posibles para tratar de precisar la identidad de los mundos, reales y virtuales a la vez, creados a lo largo de la génesis, y para definir las reglas de accesibilidad que determinan las relaciones entre esos mundos.

A crítica genética não pode ignorar a dimensão extensional da génese. Teria vantagem em se apoiar na ideia de mundo, sem dúvida mais útil, a esse respeito, que a noção de diegese, por permitir conceber uma pluralidade de mundos estipulados. Havendo cuidado em distinguir mundos textuais de mundos fictionais, podemos inspirar-nos na lógica dos mundos possíveis para tentar definir o estatuto dos mundos reais e virtuais que são criados durante a génese e para definir as regras de acessibilidade que governam as relações entre esses mundos.

La critica genetica non può ignorare la dimensione estensionale della genesi. Dovrebbe anzi fare riferimento all'idea di mondo, senza dubbio più utile in quest'ambito, rispetto alla nozione di diegesi, nella misura in cui questa permette di concepire una pluralità di mondi identificati. Distinguendo attentamente mondi testuali da mondi fittizî, si potrà prendere pretesto dalla logica dei mondi possibili per tentare di precisare lo statuto dei mondi reali o virtuali, creati nel corso del processo genetico, e per definire al contempo le regole di accessibilità che regolano i rapporti tra tali mondi. 